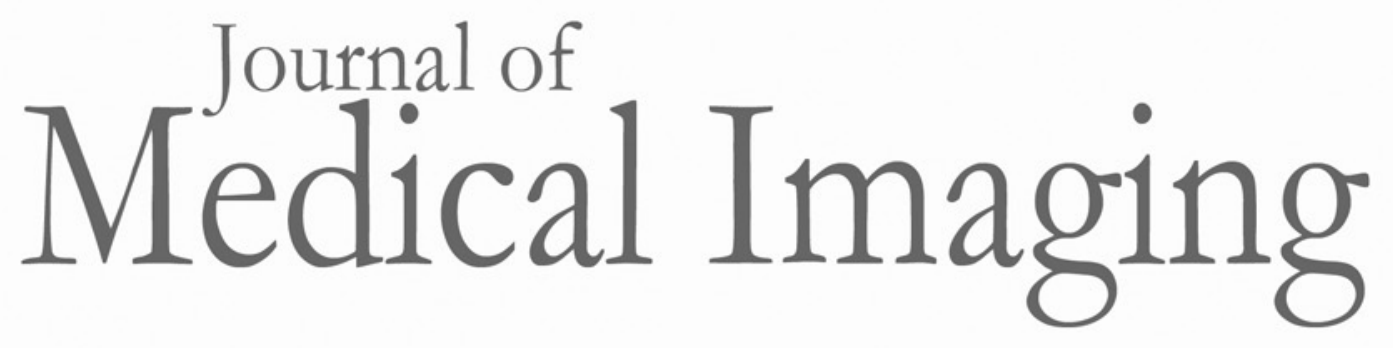

Medicallmaging.SPIEDigitalLibrary.org

\title{
In vivo reproducibility of robotic probe placement for a novel ultrasound-guided radiation therapy system
}

Muyinatu A. Lediju Bell

H. Tutkun Sen

Iulian Iordachita

Peter Kazanzides

John Wong

\section{SPIE.}




\title{
In vivo reproducibility of robotic probe placement for a novel ultrasound-guided radiation therapy system
}

\author{
Muyinatu A. Lediju Bell, ${ }^{a, *}$ H. Tutkun Sen, ${ }^{a, b}$ Iulian lordachita, ${ }^{a, c}$ Peter Kazanzides, ${ }^{a, b}$ and John Wong ${ }^{d}$ \\ a Johns Hopkins University, Laboratory for Computational Sensing and Robotics, Baltimore, Maryland 21218, United States \\ bohns Hopkins University, Department of Computer Science, Baltimore, Maryland 21218, United States \\ 'Johns Hopkins University, Department of Mechanical Engineering, Baltimore, Maryland 21218, United States \\ dJohns Hopkins University, Department of Radiation Oncology, Baltimore, Maryland 21287, United States
}

\begin{abstract}
Ultrasound can provide real-time image guidance of radiation therapy, but the probe-induced tissue deformations cause local deviations from the treatment plan. If placed during treatment planning, the probe causes streak artifacts in required computed tomography (CT) images. To overcome these challenges, we propose robot-assisted placement of an ultrasound probe, followed by replacement with a geometrically identical, CT-compatible model probe. In vivo reproducibility was investigated by implanting a canine prostate, liver, and pancreas with three 2.38-mm spherical markers in each organ. The real probe was placed to visualize the markers and subsequently replaced with the model probe. Each probe was automatically removed and returned to the same position or force. Under position control, the median three-dimensional reproducibility of marker positions was 0.6 to $0.7 \mathrm{~mm}, 0.3$ to $0.6 \mathrm{~mm}$, and 1.1 to $1.6 \mathrm{~mm}$ in the prostate, liver, and pancreas, respectively. Reproducibility was worse under force control. Probe substitution errors were smallest for the prostate $(0.2$ to $0.6 \mathrm{~mm})$ and larger for the liver and pancreas $(4.1$ to $6.3 \mathrm{~mm})$, where force control generally produced larger errors than position control. Results indicate that position control is better than force control for this application, and the robotic approach has potential, particularly for relatively constrained organs and reproducibility errors that are smaller than established treatment margins. ๑ The Authors. Published by SPIE under a Creative Commons Attribution 3.0 Unported License. Distribution or reproduction of this work in whole or in part requires full attribution of the original publication, including its DOI. [DOI: 10 .1117/1.JMI.1.2.025001]
\end{abstract}

Keywords: probe pressure; tissue deformation repeatability; mock probe; intrafraction organ motion; speckle tracking; radiation therapy.

Paper 14016PR received Feb. 18, 2014; revised manuscript received Jun. 8, 2014; accepted for publication Jun. 17, 2014; published online Jul. 23, 2014.

\section{Introduction}

Radiation therapy is a contoured approach to cancer treatment that delivers maximum radiation dosage to tumors while sparing the surrounding healthy tissue. ${ }^{1}$ Treatment planning includes a simulation computed tomography (CT) image that is used to map the expected radiation dose distribution. The treatment linear accelerator (LINAC) is then programmed to deliver the planned radiation to the patient in fractionated treatment sessions that occur over multiple days. Intrafraction organ motion due to voluntary or involuntary movement, such as patient breathing or subtle patient adjustments, decreases the effectiveness of radiation therapy, resulting in underdosage of cancerous tumors, overdosage of healthy tissue, or larger treatment margins. Although treatment margins depend on multiple factors (e.g., estimated microscopic tumor extensions, the location of nearby organs at risk, potential patient setup errors), one of the largest contributions to the planning target volume is organ motion..$^{2-4}$ As an example, intrafraction prostate motion $>2 \mathrm{~mm}$ was observed in several clinical studies, ${ }^{5,6}$ indicating that the planning target margins should at least be this large to account for motion uncertainty. Larger motion excursions were observed in the liver and pancreas. ${ }^{7,8}$ These motion studies were conducted with the implantation of radiographic markers

*Address all correspondence to: Muyinatu A. Lediju Bell, E-mail: muyinatu .ledijubell@jhu.edu and electromagnetic transponders or evaluation of four-dimensional CT images.

Real-time monitoring of tissue displacements with ultrasound (US) imaging is a noninvasive, nonionizing approach that has the potential to increase the effectiveness of radiation therapy by compensating for tumor motion during treatment. With this additional information, either radiation therapy could be halted if significant organ motion is observed or the treatment beams could be modified to move with the target. Previous work demonstrates the feasibility of real-time volumetric monitoring of intrafraction organ motion using an intrinsic property of US images known as speckle. ${ }^{9-12}$ In addition, the radiation therapy LINAC does not affect speckle tracking algorithms, ${ }^{13}$ while commercially-available optical tracking systems may be used to relate speckle tracking coordinates to the treatment room coordinate system. ${ }^{14,15}$ For subtle probe adjustments that may be required during treatment, a haptic teleoperated robotic system was introduced by Schlosser et al. ${ }^{16}$ with minimal treatment plan modifications required to accommodate the robot and US probe. Wu et al. ${ }^{17}$ additionally demonstrated that the presence of the US probe for transabdominal imaging minimally affects radiotherapy treatment plans when the anterior-posterior treatment beam is omitted.

Despite recent advances, there are two outstanding limitations to real-time monitoring with US. The primary limitation is that the tissue deformation caused by a US probe introduces a mismatch between the planned and actual radiation dose distribution. ${ }^{18-20}$ 
The US probe may be placed during treatment planning to overcome this challenge and achieve similar tissue deformations during treatment planning and delivery. However, placing the US probe at the time of treatment planning introduces the second limitation, which is that the metal components of the US probe cause streak artifacts in the CT images required for treatment planning. These artifacts may be mitigated with commercially available software; however, challenges with the software include target distortions and nonuniform removal of the metal artifacts, ${ }^{21}$ which could potentially distort CT image-based dose calculations.

To overcome the current limitations, we propose a robotassisted placement of a real US probe, followed by replacement with a geometrically identical model probe that does not contain metal components and, thus, does not cause streak artifacts in CT images. ${ }^{22,23}$ The model probe would be placed during the CT image acquisition for treatment planning, while the true US probe would be used for real-time monitoring of organ motion on each day of treatment delivery. Our previous publications introduce the cooperative control algorithm for the robotic system $^{23}$ and investigate ex vivo reproducibility of markers located at different depths from the probe. ${ }^{22}$

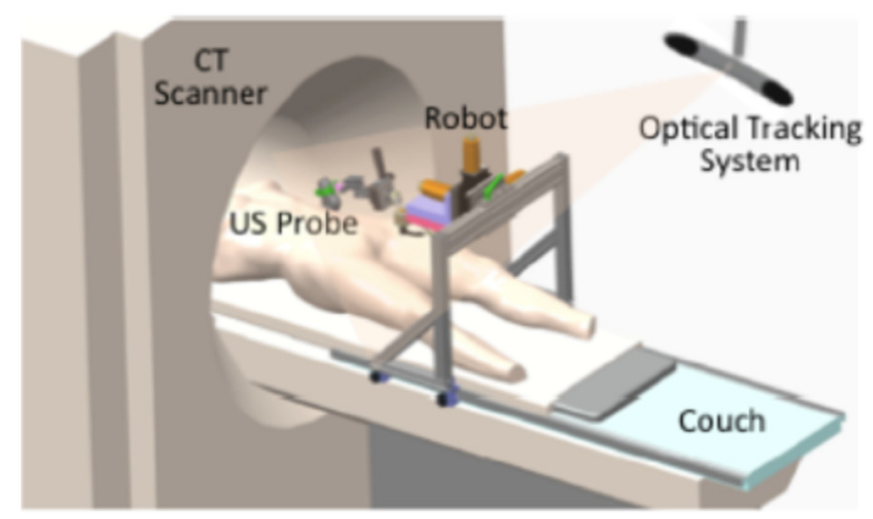

(a)
This work is the first to investigate the in vivo reproducibility of tissue deformations with robotic probe placement for guiding radiation therapy. Although probe placement is expected to deform the underlying tissue, ${ }^{19}$ we hypothesize that reproducible probe placement with robotic assistance will translate to reproducible tissue deformations in the complex in vivo environment (e.g., in the presence of multiple tissue layers with different mechanical properties, pulsating blood vessels, and organ slippage). In addition to providing repeatable positions and forces, robotic assistance has the potential to reduce the user dependency of probe placement. The twofold purpose of this study was to understand how well a robot can repeat probe-induced tissue deformations in the in vivo prostate, liver, and pancreas and to investigate tissue deformation reproducibility when substituting the real probe with the model probe in this same in vivo environment.

\section{Materials and Methods}

\subsection{Robotic System}

The proposed system for treatment planning is shown in Fig. 1(a) with a CT scanner. A similar system would be used for treatment delivery, as shown in Fig. 1(b) with a LINAC. The

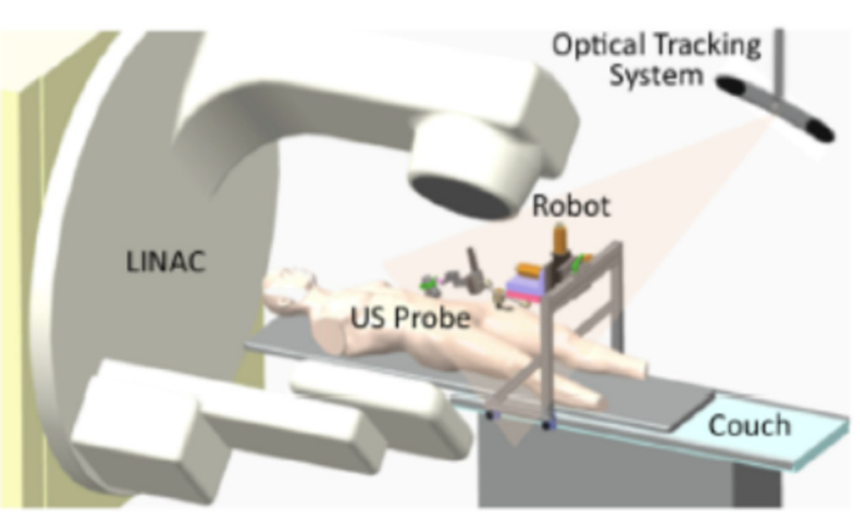

(b)

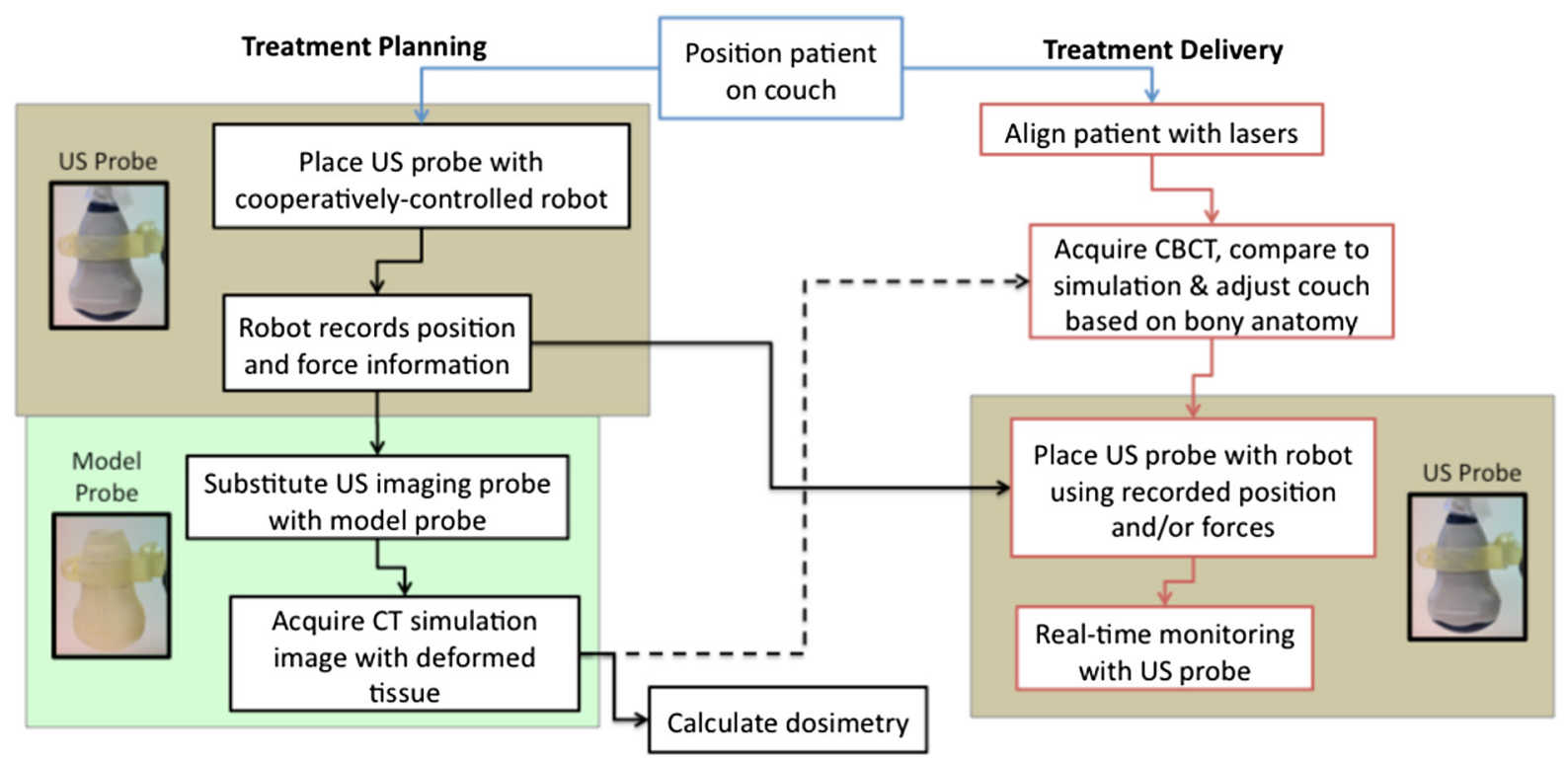

(c)

Fig. 1 Proposed robotic system for (a) treatment planning and (b) treatment delivery. (c) Corresponding workflow proposal with photographs of the real and model probes used in our study. 
robot is mounted on a bridge that rolls along the patient couch for gross adjustment and can be locked in place once a suitable position is determined. A passive arm allows the probe to extend from the bridge to the patient's organ of interest. The robot base and the end effector of the robot (i.e., the real or model probe) were equipped with optical markers for interfraction probe positioning, allowing the probe to be placed in the same orientation in the room coordinate system each day. An optical camera and the associated tracking volume for the optical tracking system are illustrated in Fig. 1. A six-axis force/torque sensor (ATI Industrial Automation, Apex, North Carolina) was mounted between the probe and passive arm to measure probe-tissue contact forces.

Probe placement was robotically controlled with three translation stages located between the bridge and passive arm. A proportional-integral-derivative controller was used to reproduce the contact force normal to the probe, such that the desired robot velocity was proportional to the difference between the desired and measured forces (i.e., zero velocity when the desired force was achieved). Assuming that the probe contact area remains the same with each placement, reproducing contact force is synonymous with reproducing probe pressure.

\subsection{Proposed Clinical Workflow}

The workflow for the proposed introduction of the robotic system is illustrated in Fig. 1(c). The color-coded shaded areas represent the novel additions facilitated by robotic assistance, where brown indicates use of the real probe and green indicates use of the model probe. Photographs of the real and model probe appear in the brown and green shaded areas, respectively. The model probe was created with a three-dimensional (3-D) printer based on dimensions of the real probe.

On simulation day, the patient would be positioned on the couch and marked with tattoos that align with the room lasers. The cooperatively controlled robot ${ }^{23}$ could then be used to place the US probe to visualize the tumor being treated. Once a suitable position is found, the robot will record the probe pose in room coordinates for repositioning on the treatment day. The contact forces will also be recorded. The real probe can then be substituted with the model probe to acquire planning CT images. The robot would be used to ensure that CT images acquired with the model probe contain the same tissue deformations that exist with the real probe, without the streak artifacts caused by the metal components of the real probe. Thus, the CT images acquired with the model probe can be used to calculate radiation dosimetry.

On each day of treatment delivery, the patient would be positioned on the couch and the room lasers will be aligned with the tattoo marks that were created at the time of simulation. The LINAC is typically equipped with an on-board cone beam $\mathrm{CT}$ (CBCT) scanner that can be used to adjust the patient based on the bony anatomy, which is not expected to change with tissue deformation. Bony anatomy is used to setup the patient because the CBCT images are of lower quality than the simulation $\mathrm{CT}$ images and are generally not sufficient to distinguish soft tissues. ${ }^{24,25}$ Alternatives to patient setup with CBCT include US-based systems, such as the Clarity Autoscan procedure for prostates (Elekta, Montreal, Canada ${ }^{26}$ and the B-Mode Acquisition and Targeting (BAT) system (NOMOS Corporation, Sewickley, Pennsylvania). ${ }^{27,28}$ In these cases, the US images can be calibrated to the same treatment and simulation room coordinate system through the use of a dedicated calibration phantom. ${ }^{14,26}$
After patient setup, another novel addition made possible by the robotic system is precise placement of the US probe in the same room coordinates on each day of treatment, assuming that conventional setup methods are sufficient to place the patient in the same position and, more importantly, place the organ at the treatment isocenter. Once the probe is in place, real-time monitoring with US can ensue during treatment delivery, as in current implementations with the Clarity Autoscan procedure for prostates. ${ }^{26}$

\subsection{Imaging Equipment}

A research-based Clarity US system was utilized to acquire volumetric B-mode ultrasound data with the Autoscan probe housed in our custom-designed probe holder. The system was connected to a Polaris optical tracking system (Northern Digital Inc., Waterloo, Canada) for monitoring the location of the US probe and robot base. CT images were acquired with a treatment planning Brilliance Big Bore 16 Slice CT scanner (Philips Medical Systems, Cleveland, Ohio).

\subsection{In Vivo Experiments}

After approval by the Johns Hopkins Animal Care and Use Committee, a canine prostate, liver, and pancreas were each implanted with three $2.38-\mathrm{mm}$ spherical metallic markers in an open-abdomen survival surgery. After one week of rest from the surgery, the dog was immobilized supine on a CT couch in a vacuum bag that is typically used to immobilize patients for treatment. The US probe was placed to visualize the implanted markers in each organ and images were acquired. The probe was automatically removed and returned to the same position (i.e., position control) at least three times for each organ, and CT images were acquired with each probe placement. This was repeated with the model probe. In addition, the model probe was removed and returned with the same force normal to the probe (i.e., force control) to investigate this alternate positioning approach. As a control experiment for each organ, three repeated CT images were acquired when no probe was in place.

US and CT images of the liver and pancreas were acquired at end inspiration to evaluate reproducibility at this phase of the respiratory cycle, which is consistent with the phase used to administer radiation treatments that require breathholds (e.g., active breathing control ${ }^{29,30}$ ). Breathholds were controlled with a mechanical ventilator. Images of the prostate were acquired under free breathing.

\subsection{Ex Vivo Experiments}

Comparative ex vivo experiments were conducted with a bovine liver partially embedded in gelatin with the liver surface exposed to air. The liver was implanted with 2.38-mm spherical metallic fiducial markers. The US probe was placed to visualize three of the implanted markers. Three markers (\#1 to 3) were placed at a distance of 1 to $2 \mathrm{~cm}$ from the tissue surface and three (\#4 to 6) were placed at a distance of 4 to $5 \mathrm{~cm}$ from the tissue surface. The deeper markers were separated from the shallower markers by $\sim 10 \mathrm{~cm}$ in the superior-inferior direction to study the effect of reproducible deformation at this distance from the imaging probe. Volumetric CT images of the fiducial markers were acquired after six repeated removals and replacements of the US probe. 


\subsection{Reproducibility Measurements}

Marker position coordinates $(x, y, z)$ in CT images of the ex vivo liver and in vivo organs were analyzed to determine the reproducibility of tissue deformations. Reproducibility was measured as the ability of the implanted markers to return to the same position. The initial marker position in each group (e.g., marker number, real probe, model probe, no probe) was taken as the baseline measurement (i.e., $x_{b}, y_{b}, z_{b}$ ), and all subsequent measurements (i.e., $x_{m}, y_{m}, z_{m}$ ) in each group were subtracted from the baseline, according to the following equation:

Reproducibility $=\sqrt{\left(x_{b}-x_{m}\right)^{2}+\left(y_{b}-y_{m}\right)^{2}+\left(z_{b}-z_{m}\right)^{2}}$.

Reproducibility results are reported in box-whisker plots with the top and bottom of each box representing the 75th and 25 th percentiles of the measurements, respectively. The line inside each box represents the median measurement, and the whiskers (i.e., lines extending above and below each box) represent the range. Outliers were defined as any value greater than 1.5 times the interquartile range and are displayed as dots.

\subsection{Displacement Measurements and Probe Substitution Error Analyses}

The difference between the mean marker positions $(\bar{x}, \bar{y}, \bar{z})$ with and without a probe in place was used to assess the similarity of tissue deformations caused by the real and model probes. Results are reported as the mean of the marker displacements in each organ, as described by the following equations:

LR Displacement $=\frac{1}{N} \sum_{m=1}^{N}\left|\bar{x}_{\text {no probe }}(m)-\bar{x}_{\text {probe }}(m)\right|$,

AP Displacement $=\frac{1}{N} \sum_{m=1}^{N}\left|\bar{y}_{\text {no probe }}(m)-\bar{y}_{\text {probe }}(m)\right|$,

SI Displacement $=\frac{1}{N} \sum_{m=1}^{N}\left|\bar{z}_{\text {no probe }}(m)-\bar{z}_{\text {probe }}(m)\right|$, where $m$ is the marker number, $N$ is the total number of markers implanted in each organ (i.e., $N=3$ ), and LR (left-right), AP (anterior-posterior), and SI (superior-inferior) refer to marker displacement along the three orthogonal directions in CT image coordinates. The standard deviations of the displacements of three markers in each organ were also calculated.

The 3-D marker displacement errors between the real and model probe (i.e., the probe substitution error) were calculated as follows for each organ:

$$
\begin{aligned}
& \text { LR Error }=\left|\bar{x}_{\text {real probe }}(m)-\bar{x}_{\text {model probe }}(m)\right|, \\
& \operatorname{APError}=\left|\bar{y}_{\text {real probe }}(m)-\bar{y}_{\text {model probe }}(m)\right|, \\
& \operatorname{SIError}=\left|\bar{z}_{\text {real probe }}(m)-\bar{z}_{\text {model probe }}(m)\right|,
\end{aligned}
$$

\section{3-D Error}

$$
=\sum_{m=1}^{N} \sqrt{\operatorname{LR} \operatorname{Error}(m)^{2}+\operatorname{APError}(m)^{2}+\operatorname{SIError}(m)^{2}},
$$

where the LR, AP, and SI errors represent the difference between the mean displacement of marker number $m$. The 3-D error described by Eq. (8) was calculated separately for the model probe under position or force control. The standard deviations of the error for the three markers were also calculated.

\section{Results}

\subsection{Ex Vivo Reproducibility}

CT images of the ex vivo liver phantom in the absence and presence of the US imaging probe are shown in Figs. 2(a) and 2(b), respectively. The circle and cross-hairs indicate the location of one of the implanted markers. Multiple CT images acquired with the US probe in place were used to determine the reproducibility of tissue deformations. The median reproducibility determined from the six implanted markers, as described by Eq. (1), ranged from 0.4 to $0.8 \mathrm{~mm}$, which is within the acceptable margins of error for radiation treatment planning for the prostate, liver, and pancreas. ${ }^{5-8,31,32}$ Figure 2(c) shows the reproducibility of the six

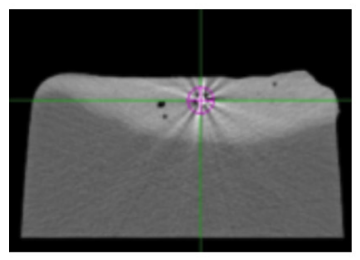

(a)

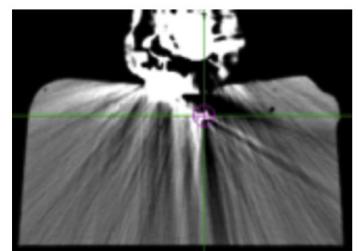

(b)

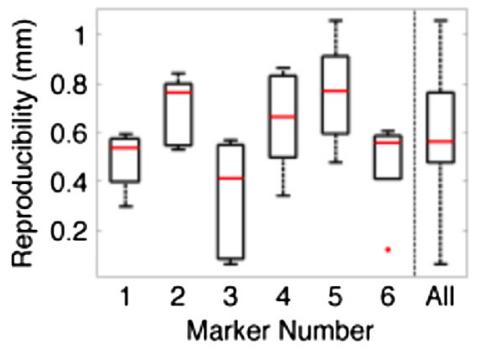

(c)

Fig. 2 CT images of the ex vivo phantom in the (a) absence and (b) presence of the ultrasound (US) probe. The US probe causes streak artifacts in CT images, yet it was still possible to discern marker locations, as indicated by the circles and cross-hairs. (c) Reproducibility of the six markers implanted in the ex vivo liver tissue. 
markers individually as well as all six markers combined. The combined approach was used to report reproducibility in vivo. An outlier appears at $0 \mathrm{~mm}$ for marker number 6 . In addition, the bottom whisker for this marker coincides with the 25 th percentile. The mean of all measured contact forces \pm one standard deviation was $1.3 \pm 0.1 \mathrm{~N}$.

\subsection{In Vivo Reproducibility}

\subsubsection{Prostate}

US and CT images of the prostate are shown in Figs. 3(a) to 3(c). The arrow in Fig. 3(a) points to one of the implanted markers, as confirmed with a 3-D ultrasound scan of the prostate and the characteristic comet-tail artifact produced by the metal marker. The green circle in the CT image of Fig. 3(b) indicates the location of one of the markers in the dog's prostate. Although there were streak artifacts due to the presence of the real probe, it was possible to identify the marker and determine its 3-D position coordinates from CT images. Figure 3(c) shows a similar CT slice with the model probe in place. The streak artifacts caused by the real probe are not present in the CT image with the model probe.

Figure 3(d) displays the recorded 3-D marker positions, as analyzed from the acquired CT images. There is a pronounced distinction between the three markers with and without the probe in place. Tissue deformations were reproducible with a median of $0.6 \mathrm{~mm}$ with the real probe under position control. When the model probe was under position and force control, the median reproducibility was 0.7 and $1.2 \mathrm{~mm}$, respectively, as indicated in Fig. 3(e). These values are comparable to the control experiment with no probe in place (median 3-D reproducibility of $0.4 \mathrm{~mm}$ ) and to marker reproducibility measured in the corollary ex vivo experiment.

\subsubsection{Liver}

The liver was imaged using a subcostal US window. The US image in Fig. 4(a) shows two of the implanted markers (arrows) as well as the gall bladder. The corresponding CT images of the implanted markers do not show the US probe because it is not in the same axial CT slice as the markers, as illustrated in Fig. 4(b), showing one of the implanted markers (circle). Note that streak artifacts due to the presence of the US probe did not appear in the axial CT slices surrounding the markers when the probe was placed subcostally.

The marker positions in the liver measured with and without the real and model US probes are shown in Fig. 4(c). The marker positions are more scattered without the probe in place [i.e., three distinct markers are not easily discernible, unlike Fig. 3(d)], indicating that the presence of the probe helps to stabilize marker positions in the liver. A complementary 3-D rotational view of the marker positions is available in Video 1.

The median 3-D reproducibility of marker positions, shown in Fig. 4(d), measured $0.3 \mathrm{~mm}$ with the real probe and $0.6 \mathrm{~mm}$ with the model probe returning to the same position. These values are within acceptable treatment margins and significantly less than the control with no probe in place (median reproducibility of $4.7 \mathrm{~mm}$ ). When the model probe was returned to the same initial force as the real probe, the reproducibility was poor (median $14 \mathrm{~mm}$ ).

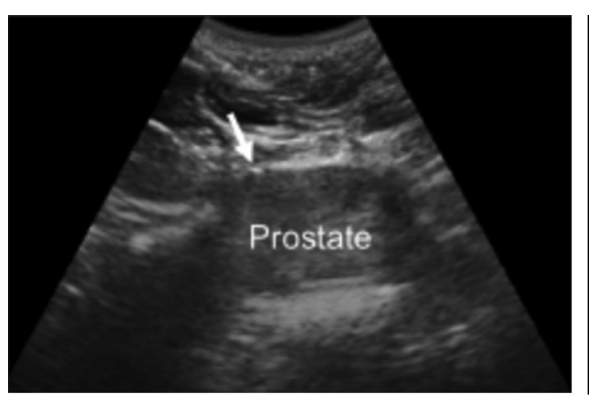

(a)

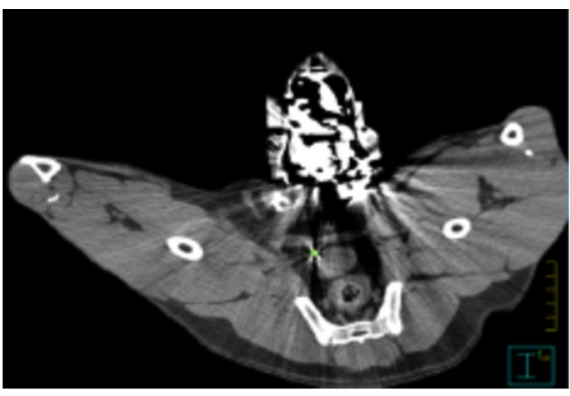

(b)

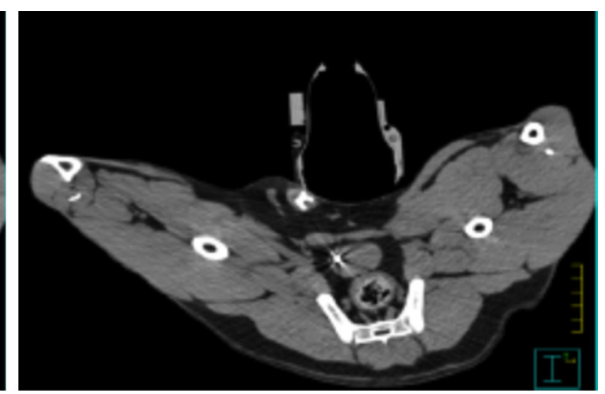

(c)

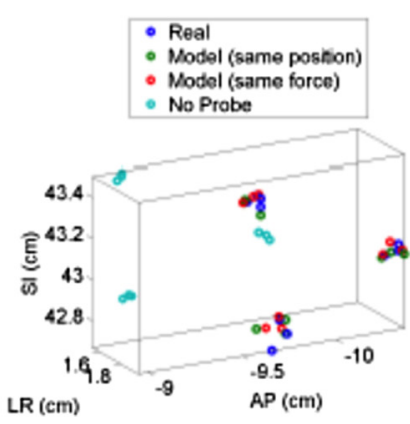

(d)

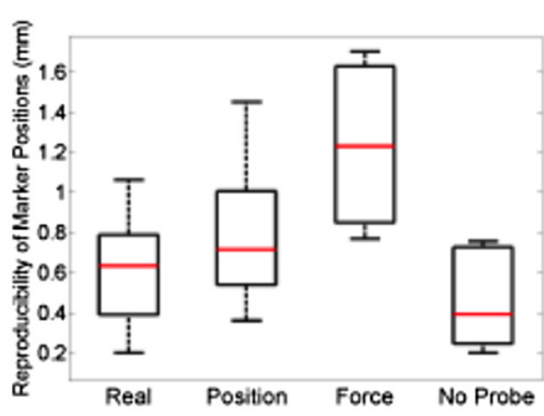

(e)

Fig. 3 (a) US image of the prostate and corresponding axial CT images of the dog prostate with the (b) real probe and (c) model probe in place. (d) Prostate marker positions recorded from CT images. Each cluster in each probe category (e.g., real probe, model probe, no probe) represents the different spatial positions of one of the three implanted markers after repeated trials. (e) Reproducibility of marker positions after repeated robotic placement with the real probe, model probe after returning to the same position or force, and when no probe was in place, from left to right, respectively. 


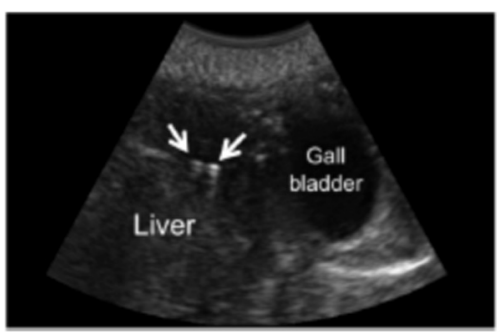

(a)

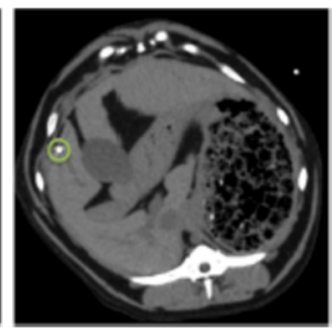

(b)

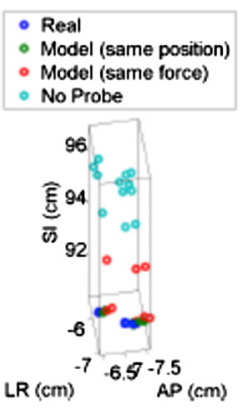

(c)

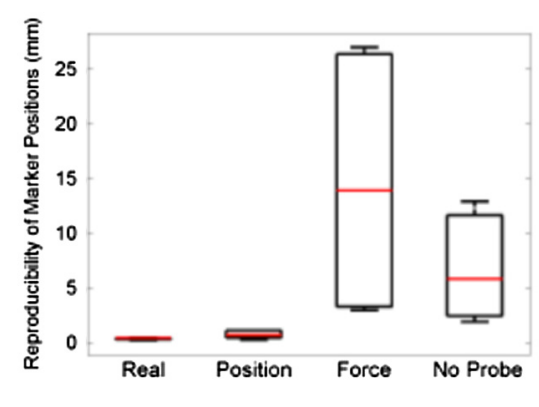

(d)
Fig. 4 (a) US and (b) axial CT images of markers implanted in the liver. (c) Liver marker positions recorded from CT images (See Video 1, MPEG, 1.25 MB [URL: http://dx.doi.org/10.1117/1.JMI.1.2 .025001.1] for a 3-D rotational view). (d) Reproducibility of marker positions after repeated robotic placement with the real probe, model probe after returning to the same position or force, and when no probe was in place, from left to right, respectively.

\subsubsection{Pancreas}

The pancreas was poorly visualized with US, but the CT image was used to confirm that the probe was placed in a location that would displace the implanted markers, as shown in Fig. 5(a). Two of the implanted markers are circled on the CT image. The marker positions in the pancreas measured with and without the real and model US probes are shown in Fig. 5(b) and in the related Video 2. The median 3-D reproducibility of marker positions is shown in Fig. 5(c), which measured 1.6 and $1.1 \mathrm{~mm}$ with the real and model probes, respectively, under position control. Although an outlier appears at $0 \mathrm{~mm}$ for the model probe under position control, these median values are comparable to the

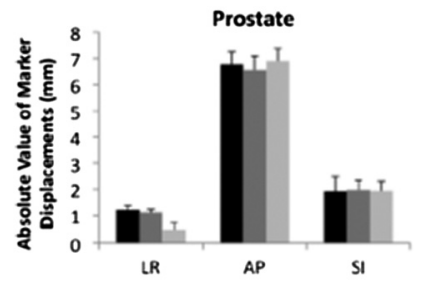

(a)

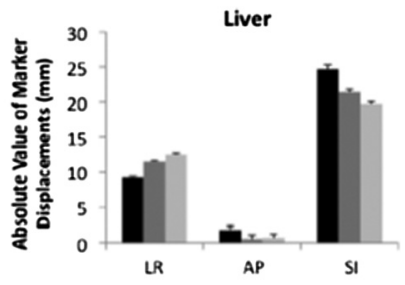

(b)

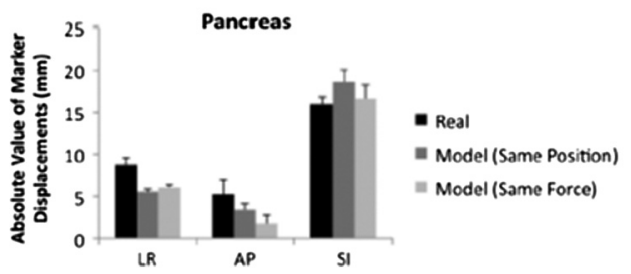

(c)

Fig. 6 Comparison between marker displacements with real and model probes for the (a) prostate, (b) liver, and (c) pancreas. The prostate appears to be constrained in the superior-inferior direction, the liver appears to be drifting with time, and the pancreas appears to move freely under the applied probe pressure.

control with no probe, which had a median reproducibility of $1.3 \mathrm{~mm}$. The median 3-D reproducibility was worse $(4.3 \mathrm{~mm})$ under force control.

\subsection{Marker Displacements and Probe Substitution Errors}

Figure 6 illustrates the marker displacements due to placement of the real and model probes, as calculated by Eqs. (2) to (4). For the prostate [Fig. 6(a)], the largest marker displacements $(\sim 7 \mathrm{~mm})$ occurred in the AP direction, which was normal to the direction of the US beams. The displacements are most similar in the SI direction $(\sim 2 \mathrm{~mm})$, indicating that the prostate is potentially constrained in this direction. The mean 3-D error between real and model probes was $0.3 \mathrm{~mm}$ under position control and $0.7 \mathrm{~mm}$ under force control. These probe substitution errors are within the acceptable margins for treating prostates. The mean of all measured contact forces \pm one standard deviation was $10.5 \pm 0.3 \mathrm{~N}$, while the measured forces under force control

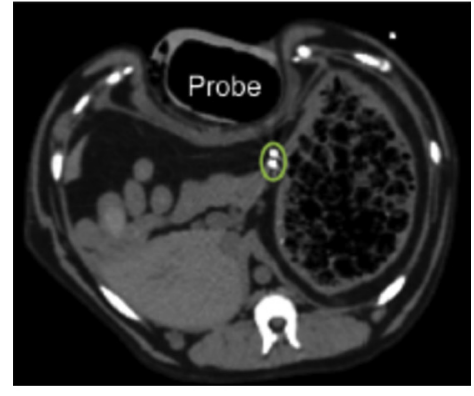

(a)

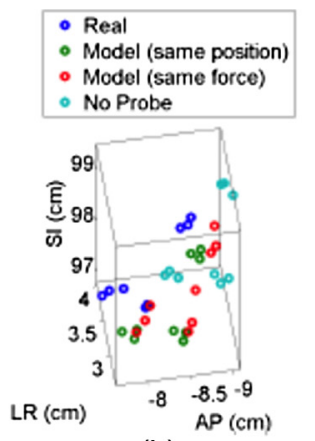

(b)

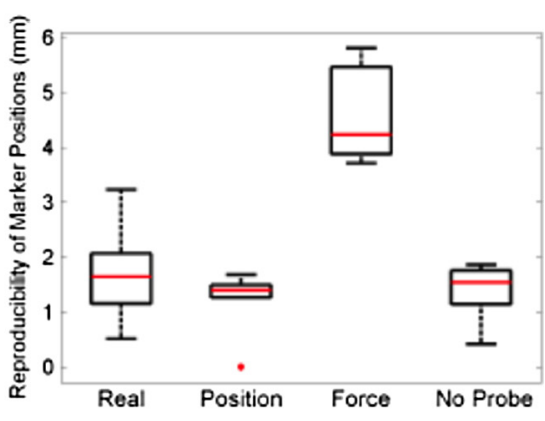

(c)

Fig. 5 (a) Axial CT image of the pancreas and two implanted markers (circled). (b) Pancreas marker positions recorded from CT images (see Video 2, MPEG, 1.67 MB [URL: http://dx.doi.org/10.1117/1 .JMI.1.2.025001.2] for a 3-D rotational view). (c) Reproducibility of marker positions after repeated robotic placement with the real probe, model probe after returning to the same position or force, and when no probe was in place, from left to right, respectively. 
Table 1 Summary of contact forces and three-dimensional (3-D) probe substitution errors reported as the mean \pm one standard deviation.

\begin{tabular}{lcccc} 
Organ & $\begin{array}{c}\text { All contact force } \\
\text { measurements }(\mathrm{N})\end{array}$ & $\begin{array}{c}\text { Force-controlled } \\
\text { contact force }(\mathrm{N})\end{array}$ & $\begin{array}{c}\text { 3-D error with position-controlled } \\
\text { model probe }(\mathrm{mm})\end{array}$ & $\begin{array}{c}\text { 3-D error with force-controlled } \\
\text { model probe }(\mathrm{mm})\end{array}$ \\
\hline Prostate & $10.5 \pm 0.3$ & $10.5 \pm 0.1$ & $0.3 \pm 0.1$ & $0.7 \pm 0.1$ \\
Liver & $34.5 \pm 2.4$ & $37.1 \pm 1.3$ & $4.1 \pm 0.3$ & $6.3 \pm 0.5$ \\
Pancreas & $34.5 \pm 1.2$ & $34.8 \pm 0.8$ & $4.7 \pm 0.7$ & $4.6 \pm 0.7$ \\
\hline
\end{tabular}

had a smaller standard deviation $(0.1 \mathrm{~N})$. These probe substitution errors and measured forces are summarized in Table 1.

The largest marker displacements in the liver $(\sim 25 \mathrm{~mm})$ occurred in the SI direction, as shown in Fig. 6(b). This direction is primarily aligned with the direction of the US beams for the subcostal probe orientation. Note that the displacements in the LR and SI directions progressively increase or decrease with time (i.e., from placement of the real probe to placement of the model probe with force control), indicating that there is a velocity vector in these directions. This is also evident from Video 1, which shows the liver marker positions related to Fig. 4(c). The mean 3-D error between the real and model probes for the liver was $4.1 \mathrm{~mm}$ with position control and $6.3 \mathrm{~mm}$ with force control. The mean of all measured contact forces \pm one standard deviation was $34.5 \pm 2.4 \mathrm{~N}$. The measured contact forces under force control was $37.1 \pm 1.3 \mathrm{~N}$. These results are summarized in Table 1 .

There is no clear spatial or temporal trend in marker displacement measurements for the pancreas, as shown in Fig. 6(c). The mean 3-D error between the real and model probes was $4.7 \mathrm{~mm}$ with position control and $4.6 \mathrm{~mm}$ with force control. The mean of all measured contact forces \pm one standard deviation was $34.5 \pm 1.2 \mathrm{~N}$. The measured contact forces under force control was $34.8 \pm 0.8 \mathrm{~N}$. These results are summarized in Table 1 .

\section{Discussion}

Position control was more reproducible than force control in the three organs studied, indicating that returning to the same position is the preferred method for substituting the real probe with the model probe to acquire simulation CT images. Under force control, the reproducibility of marker displacements was poorer, particularly for the liver and pancreas, although the contact forces were reproduced with low standard deviations ( 0.1 to $1.3 \mathrm{~N}$ ) in the three organs studied. The poorer reproducibility with force control is likely due to the nonlinear relationship between force and displacement in biological tissues. ${ }^{33,34}$ Given this nonlinearity, reproducing force or probe pressure does not necessarily place the organ in the same location as reproducing probe position. This observation is further supported by the results reported in Table 1, which indicate that the larger standard deviations of all measured forces for the liver and prostate are caused by large variations in forces measured under position control. The larger force variations under position control are likely caused by tissue nonlinearities and varying internal forces in vivo (e.g., pulsating blood vessels and tissue preconditioning with repeated probe placement ${ }^{33}$ ).

Out of the three organs studied, position control reproducibility was poorest for the pancreas (medians of 1.6 and $1.1 \mathrm{~mm}$ with the real and model probes, respectively) with two potential causes. First, the contact force was larger $(\sim 34 \mathrm{~N})$, and we suspect that smaller forces may be suitable to image humans. Nonetheless, similar contact forces were used to image the liver and good reproducibility was achieved with position control. Thus, the more likely explanation for the poorer reproducibility is that the pancreas is less constrained than the prostate, and it has more spatial freedom both when responding to the induced probe pressure and when no probe was present. In addition, the probe substitution errors in three directions (e.g., $4.7 \pm$ $0.7 \mathrm{~mm}$ with position control) was smaller than the measured pancreas excursions in one direction (e.g., 6 to $10 \mathrm{~mm})^{8}$ and common treatment margins in one direction (e.g., $1 \mathrm{~cm}){ }^{32} \mathrm{It}$ is promising that these errors are smaller than existing treatment margins, which are primarily derived from the expected organrelated positional uncertainty. The inclusion of US imaging can reduce this uncertainty with its ability to visualize soft tissues and monitor organ motion. Therefore, the proposed system has the potential to reduce existing treatment margins to the smaller errors measured with robot-assisted placement of the US probe.

While the liver had good reproducibility under position control (median of 0.3 and $0.6 \mathrm{~mm}$ with the real and model probes, respectively), the probe substitution errors were larger with position control $(4.1 \pm 0.3 \mathrm{~mm})$. However, a model probe is not necessary for the subcostal acoustic window due to the absence of streak artifacts. In addition to more spatial freedom compared to the prostate, natural drifting of the liver ${ }^{35}$ could be responsible for the appearance of a velocity vector in the LR and SI directions [Fig. 6(b)] and the large variations in marker positions when no probe was present [Fig. 4(c)]. When comparing position control reproducibility results with reproducibility when no probe was in place, the probe seems to act as an abdominal compressor $^{36}$ that stabilizes the liver position.

When translated to human patients, pancreas imaging within the current $1 \mathrm{~cm}$ treatment margins ${ }^{32}$ and transabdominal prostate imaging with less experienced users ${ }^{37}$ might benefit from the proposed robotic system. In addition, current implementations of transperineal US imaging does not interfere with the treatment beams, ${ }^{26}$ which is similarly true when placing the probe for a subcostal view of the liver. Thus, a robotic system that implements subcostal liver imaging with no probe substitution might also be considered in humans, and it is ideal for organ monitoring with US because the axial dimension of the US probe (i.e., the direction where speckle tracking is best) aligns with the largest direction of expected motion. ${ }^{9,11}$ Transabdominal gynecological imaging could additionally benefit from a robotic system, based on the probe-induced deformations and user-dependent reproducibility results achieved in a gynecological phantom and in five cervical cancer patients. ${ }^{38,39}$ The required probe pressure, existing treatment margins, and spatial constraints of the organ being treated should be investigated to confidently determine the clinical utility of the robotic system in each potential application. 
The ex vivo results in Fig. 2 provide a lower-bound estimate of reproducibility in vivo. They complement our previous ex vivo studies using the same phantom to report $0.3 \mathrm{~mm}$ mean absolute difference between marker displacements before and after repeated probe placement with position control. ${ }^{22}$ In addition, the ex vivo study herein differs from the previous study because it considers the reproducibility of markers that are $10 \mathrm{~cm}$ from the probe and, thus, not visualized in the US image.

Measurements of interfraction probe placement and the associated deformation reproducibility were beyond the scope of this paper, however, the presented results can be considered as "best case" scenarios for in vivo organs that are similar to the organs studied. Position-controlled probe placement on different treatment days could be achieved with an optical tracking system that records the probe pose in the room coordinate system during treatment planning. The saved probe position and orientation would then be recreated on each day of treatment. For scenarios where it is not feasible to reproduce the probe position (e.g., in cases with large day-to-day patient setup errors, anatomy changes, or relatively unconstrained organs), alternative methods might be necessary. For example, US image information could be used to adjust the couch and place the organ at the treatment isocenter, ${ }^{40}$ as is currently implemented with the Clarity $^{26,41}$ and BAT $^{27,28}$ US-based systems. In these cases, a robot could reduce interuser variability and reproduce simulation day probe-induced deformations by using image feedback or visual servoing to control probe placement (e.g., based on landmarks in the US image acquired on the simulation day). The results herein suggest that this type of position control would yield more reproducible organ deformation than force or pressure control if small probe adjustments are needed during treatment delivery. Given these alternative approaches, the overall goal of using ultrasound to monitor tumor motion would remain unchanged.

\section{Conclusions}

Robotic placement of real and model probes were generally reproducible for constrained in vivo organs, such as the prostate, and less reproducible for organs with more spatial freedom (e.g., the pancreas). For the prostate, the model probe was capable of reproducing the in vivo displacements introduced by the real probe with minimal 3-D error. Less error was produced with position control rather than force control, indicating that position control should be used for the probe substitution. Results are promising for utilizing the proposed robotic US system to monitor organ motion in real time during radiation therapy, particularly if minimal probe pressure is used to visualize the tumor and the error introduced by the probe substitution is smaller than the existing treatment margins.

\section{Acknowledgments}

This work is supported in part by NIH R01 CA161613. M. A. Lediju Bell is a recipient of the Ford Foundation and UNCF/ Merck Postdoctoral Research Fellowships.

\section{References}

1. F. M. Khan, The Physics of Radiation Therapy, Vol. 3, Lippincott Williams \& Wilkins, Philadelphia (2003).

2. J. A. Purdy, "Current ICRU definitions of volumes: limitations and future directions," in Seminars in Radiation Oncology, Vol. 14, pp. 27-40, Elsevier (2004).
3. J. C. Stroom and B. J. M. Heijmen, "Geometrical uncertainties, radiotherapy planning margins, and the ICRU-62 report," Radiother. Oncol. 64(1), 75-83 (2002).

4. M. Van Herk, "Errors and margins in radiotherapy," in Seminars in Radiation Oncology, Vol. 14, pp. 52-64, Elsevier (2004).

5. A. N. Kotte et al., "Intrafraction motion of the prostate during externalbeam radiation therapy: analysis of 427 patients with implanted fiducial markers," Int. J. Radiat. Oncol. Biol. Phys. 69(2), 419-425 (2007).

6. K. R. Britton et al., "Evaluation of inter-and intrafraction organ motion during intensity modulated radiation therapy (IMRT) for localized prostate cancer measured by a newly developed on-board image-guided system," Radiat. Med. 23(1), 14-24 (2005).

7. D. P. Gierga et al., "Quantification of respiration-induced abdominal tumor motion and its impact on IMRT dose distributions," Int. J. Radiat. Oncol. Biol. Phys. 58(5), 1584-1595 (2004).

8. S. D. Goldstein et al., "Use of respiratory-correlated four-dimensional computed tomography to determine acceptable treatment margins for locally advanced pancreatic adenocarcinoma," Int. J. Radiat. Oncol. Biol. Phys. 76(2), 597-602 (2010).

9. M. A. Lediju Bell et al., "In vivo liver tracking with a high volume rate 4D ultrasound scanner and a 2D matrix array probe," Phys. Med. Biol. 57(5), 1359 (2012).

10. J. M. Rubin et al., "Potential use of ultrasound speckle tracking for motion management during radiotherapy preliminary report," $J$. Ultrasound Med. 31(3), 469-481 (2012).

11. E. J. Harris et al., "Speckle tracking in phantom and feature-based tracking in liver in the presence of respiratory motion using $4 \mathrm{D}$ ultrasound," Phys. Med. Biol. 55(12), 3363-3380 (2010).

12. T. P. O'Shea et al., " $4 \mathrm{D}$ ultrasound speckle tracking of intra-fraction prostate motion: a phantom-based comparison with x-ray fiducial tracking using cyberknife," Phys. Med. Biol. 59(7), 1701 (2014).

13. A. Hsu et al., "Feasibility of using ultrasound for real-time tracking during radiotherapy," Med. Phys. 32(6), 1500-1512 (2005).

14. L. G. Bouchet et al., "Calibration of three-dimensional ultrasound images for image-guided radiation therapy," Phys. Med. Biol. 46(2), 559-578 (2001).

15. S. L. Meeks et al., "Optically guided patient positioning techniques," in Seminars in Radiation Oncology, Vol. 15, pp. 192-201, Elsevier (2005).

16. J. Schlosser, K. Salisbury, and D. Hristov, "Telerobotic system concept for real-time soft-tissue imaging during radiotherapy beam delivery," Med. Phys. 37(12), 6357-6367 (2010).

17. J. Wu et al., "Effect of ultrasound probe on dose delivery during realtime ultrasound-guided tumor tracking," in 28th Annual Int. Conf. of the IEEE Engineering in Medicine and Biology Society, pp. 3799-3802, IEEE (2006).

18. M. Fargier-Voiron et al., "Impact of probe pressure variability on prostate localization for ultrasound-based image-guided radiotherapy," Radiother. Oncol. 111(1), 132-137 (2014).

19. C. F. Serago et al., "Initial experience with ultrasound localization for positioning prostate cancer patients for external beam radiotherapy," Int. J. Radiat. Oncol. Biol. Phys. 53(5), 1130-1138 (2002).

20. X. Artignan et al., "Online ultrasound image guidance for radiotherapy of prostate cancer: impact of image acquisition on prostate displacement," Int. J. Radiat. Oncol. Biol. Phys. 59(2), 595-601 (2004).

21. R. Al-Senan and M. Yester, "Metal artifact redcution in ct for surgical screws: comparison between two commercially available software packages," Med. Phys. 40(6), 129-129 (2013).

22. M. A. Lediju Bell et al., "Repeatability of robotic placement of ultrasound probes for an integrated US-CT approach to image-guided radiotherapy," Med. Phys. 40(6), 376-376 (2013).

23. H. T. Sen et al., "A cooperatively controlled robot for ultrasound monitoring of radiation therapy," IEEE/RSJ Int. Conf. on Intelligent Robots and Systems, pp. 3071-3076 (2013).

24. O. Gayou and M. Miften, "Commissioning and clinical implementation of a mega-voltage cone beam ct system for treatment localization," Med. Phys. 34(8), 3183-3192 (2007).

25. J. A. Snir et al., "Evaluation of inter-fraction prostate motion using kilovoltage cone beam computed tomography during radiotherapy," Clin. Oncol. 23(9), 625-631 (2011).

26. M. Lachaine and T. Falco, "Intrafractional prostate motion management with the Clarity Autoscan system," Med. Phys. Int. 1(1), 72-80 (2013). 
27. K. M. Langen et al., "Evaluation of ultrasound-based prostate localization for image-guided radiotherapy," Int. J. Radiat. Oncol. Biol. Phys. 57(3), 635-644 (2003).

28. A. Chandra et al., "Experience of ultrasound-based daily prostate localization," Int. J. Radiat. Oncol. Biol. Phys. 56(2), 436-447 (2003).

29. J. W. Wong et al., "The use of active breathing control $(\mathrm{ABC})$ to reduce margin for breathing motion," Int. J. Radiat. Oncol. Biol. Phys. 44(4), 911-919 (1999).

30. L. A. Dawson et al., "The reproducibility of organ position using active breathing control (ABC) during liver radiotherapy," Int. J. Radiat. Oncol. Biol. Phys. 51(5), 1410-1421 (2001).

31. B. Engels et al., "Conformal arc radiotherapy for prostate cancer: increased biochemical failure in patients with distended rectum on the planning computed tomogram despite image guidance by implanted markers," Int. J. Radiat. Oncol. Biol. Phys. 74(2), 388-391 (2009).

32. J. D. Murphy et al., "Full-dose gemcitabine and concurrent radiotherapy for unresectable pancreatic cancer," Int. J. Radiat. Oncol. Biol. Phys. 68(3), 801-808 (2007).

33. B. K. Tay, J. Kim, and M. A. Srinivasan, "In vivo mechanical behavior of intra-abdominal organs," IEEE Trans. Biomed. Eng. 53(11), 21292138 (2006).

34. F. Pervin, W. W. Chen, and T. Weerasooriya, "Dynamic compressive response of bovine liver tissues," J. Mech. Behav. Biomed. Mater. 4(1), 76-84 (2011).

35. H. Shirato et al., "Intrafractional tumor motion: lung and liver," in Seminars in Radiation Oncology, Vol. 14, pp. 10-18, Elsevier (2004).

36. J. H. Heinzerling et al., "Four-dimensional computed tomography scan analysis of tumor and organ motion at varying levels of abdominal compression during stereotactic treatment of lung and liver," Int. J. Radiat. Oncol. Biol. Phys. 70(5), 1571-1578 (2008).

37. D. Robinson et al., "An evaluation of the clarity 3D ultrasound system for prostate localization," J. Appl. Clin. Med. Phys. 13(4), 100-112 (2012).

38. M. Baker, J. A. Jensen, and C. F. Behrens, "Inter-operator variability in defining uterine position using three-dimensional ultrasound imaging," in IEEE Int. Ultrasonics Symp., pp. 848-851, IEEE (2013).

39. M. Baker, J. A. Jensen, and C. F. Behrens, "Determining inter-fractional motion of the uterus using 3D ultrasound imaging during radiotherapy for cervical cancer," Proc. SPIE, 9040, 90400 Y (2014).

40. J. Troccaz et al., "Conformal external radiotherapy of prostatic carcinoma: requirements and experimental results," Radiother. Oncol. 29(2), 176-183 (1993).
41. S. van der Meer et al., "Critical assessment of intramodality 3D ultrasound imaging for prostate IGRT compared to fiducial markers," Med. Phys. 40(7), 071707 (2013).

Muyinatu A. Lediju Bell is a postdoctoral fellow at Johns Hopkins University. She earned her BS degree in mechanical engineering from the Massachusetts Institute of Technology in 2006 and her PhD degree in biomedical engineering from Duke University in 2012. Her research interests include enhancing ultrasound and photoacoustic image quality and integrating imaging with robotics to improve the detection and treatment of cancer.

H. Tutkun Sen has been a PhD student in the Computer Science Department at Johns Hopkins University since 2010. He has double bachelor degrees in mechanical engineering and electrical electronics engineering from Middle East Technical University, Turkey. His research interests include medical robotics, surgical navigation systems, and sensor fusion algorithms.

lulian lordachita received his BEng degree in mechanical engineering, MEng degree in industrial robots, and $\mathrm{PhD}$ degree in mechanical engineering from the University of Craiova, Romania, in 1984, 1989, and 1996, respectively. He is currently an assistant research professor in the Department of Mechanical Engineering at Johns Hopkins University, where he is engaged in research on medical robotics. His current research interests include design and manufacturing of surgical instrumentation and medical robots.

Peter Kazanzides received his $\mathrm{PhD}$ degree in electrical engineering from Brown University and started working on surgical robotics in 1989 as a postdoctoral researcher at the IBM T.J. Watson Research Center. He cofounded Integrated Surgical Systems in 1990 to commercialize the ROBODOC System for orthopaedic surgery and served as the director of robotics and software. He joined Johns Hopkins University in 2002 and is currently an associate research professor of computer science.

John Wong is a professor and director of medical physics in radiation oncology. He has played principal and active roles on numerous public and industrial research initiatives. $\mathrm{He}$ is an inventor of the clinical Active Breathing Coordinator and the Cone-Beam Computed Tomography systems, as well as the image guidance platform for preclinical radiation research. His current research focus includes robotic ultrasound guidance for radiotherapy, preclinical molecular imaging, and informatics infrastructure for decision support. 